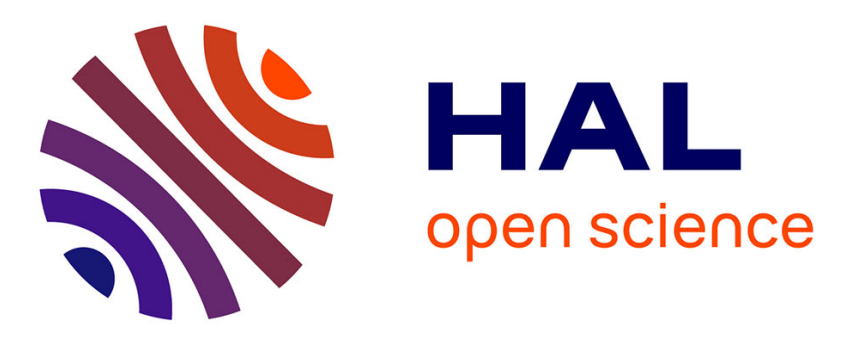

\title{
Vehicular Communications in Tunnels using VLC
}

Edmundo Torres-Zapata, Victor Guerra, Jose Rabadan, Rafael Perez-Jimenez, Jose Martin Luna-Rivera

\section{To cite this version:}

Edmundo Torres-Zapata, Victor Guerra, Jose Rabadan, Rafael Perez-Jimenez, Jose Martin LunaRivera. Vehicular Communications in Tunnels using VLC. 15th International Conference on Telecommunications (ConTEL 2019), Jul 2019, Graz, Austria. 10.1109/ConTEL.2019.8848500 . hal02563829

\section{HAL Id: hal-02563829 \\ https://hal.science/hal-02563829}

Submitted on 5 May 2020

HAL is a multi-disciplinary open access archive for the deposit and dissemination of scientific research documents, whether they are published or not. The documents may come from teaching and research institutions in France or abroad, or from public or private research centers.
L'archive ouverte pluridisciplinaire HAL, est destinée au dépôt et à la diffusion de documents scientifiques de niveau recherche, publiés ou non, émanant des établissements d'enseignement et de recherche français ou étrangers, des laboratoires publics ou privés. 


\title{
Vehicular Communications in Tunnels using VLC
}

\author{
Edmundo Torres-Zapata, Victor Guerra, Jose Rabadan \\ and Rafael Perez-Jimenez \\ IDeTIC, Universidad de Las Palmas de Gran Canaria \\ Las Palmas de Gran Canaria, Spain \\ \{etorres,vguerra,jrabadan,rperez\}@idetic.eu \\ Jose Martin Luna-Rivera \\ Facultad de Ciencias, Universidad Autonoma de San Luis Potosi \\ San Luis Potosi, Mexico \\ mlr@uaslp.mx
}

\begin{abstract}
The advances in vehicular communications and Internet of Things (IoT) technologies have provided a promising opportunity to further address the increasing Intelligent Transport System (ITS) issues. In general, ITS coverage in closed spaces is increasingly seen as essential, particularly in road tunnels, where reliable systems are needed. Visible light communication appears as a potential alternative to Radio Frequency (RF) technologies for road tunnels where RF signals face some constraints that severely affect its propagation performance. In this paper we present a VLC communication infrastructure for vehicles taking into consideration the different elements of road tunnels. A feasibility analysis of the proposed system is also shown.
\end{abstract}

Index Terms - Visible Light Communication, Vehicular Communications, Internet of Things

\section{INTRODUCTION}

In the last few years, Internet of Things (IoT) has covered a vast number of application fields in peoples daily life, as it improves the performance of existing processes and opens the possibility of offering new services. Vehicular industry is one of the fields which are already taking advantage of IoT, and this sector is planning to massively adopt this technology. The main application of IoT in a vehicular environment is Intelligent Transport Systems (ITS), providing the car the ability to get information from the environment [1], avoiding risks, decreasing the damage in case of an accident happens, improving the driving experience, etc. This information is not only obtained from the vehicles on-board sensors, but also gathered from other vehicles and external sensors on street facilities, or even provided by the local administration. Vehicular communication allows information feedback to other users regarding any anomaly detected by the car. This application scenario creates the necessity of communication between vehicles.

Dedicated Short Range Communication (DSRC) is one of the primary areas of research and development for vehicular communication, which operates in the $5 \mathrm{GHz}$ frequency band [2]. DSRC presents several issues such as high latency when

This project has received funding from the European Unions Horizon 2020 research and innovation programme under the Marie Skodowska-Curie grant agreement No 764461 it is establishing communication with new networks elements, and the system needs additional solutions to locate the vehicle. The mentioned disadvantages has considerable importance in the emergency application, when the communication system needs to communicate with one specific user, which it is located in the emergency area. One option, which does not require positioning system, is increasing the complexity of the Medium Access Control (MAC) layer. Unfortunately, this action increases the time consumption to complete the task.

Additionally, the overall performance of the system decreases when the vehicle is transmitting warning messages, in a harsh environment using DSCR. RF signal propagation in tunnels exhibits significant differences from outdoor environments, where the communication channel rapidly degrades. This offers an opportunity for other technologies to take place in these environments. Several previous studies about radio propagation in tunnels have been carried out, but only a few of them have taken into consideration the impact of the traffic in the communication channel. In [3], it was shown that delay spread increases considerably when the tunnel has traffic, compared with the "no traffic" situation. This delay spread is negligible when no obstacles are present, but in the presence of static obstacles it becomes around $0.2 \mu \mathrm{s}$, showing a linear dependence with distance. Furthermore, when there are vehicles the delay spread increases up to $0.5 \mu \mathrm{s}$, decreasing the achievable data rate.

A realistic evaluation of communications inside a tunnel at frequencies around $2.5 \mathrm{GHz}$ was performed in [4], showing that jitter values can reach up to $0.22 \mu \mathrm{s}$ and the data rate can diminish up to $52.4 \%$ in the worst case. The stationarity of the channel in these environments was addressed in [5], establishing the coherence time and showing the dependence on the relative speed difference of both transmitter and receiver vehicles (which generates Doppler spread).

Visible Light Communications (VLC) appears as an available communication technology on these environments. Since it does not interfere with other RF-based wireless technologies, it can be used in a complementary way. Despite the short range and the line-of-sight (LOS) requirements, which limits 
its application to neighbor vehicles and close infrastructure endpoints, vehicular VLC is especially useful when sending warning messages. Moreover, as VLC signals are IM/DD (Intense Modulated, directly detected), the frequency shift produced by the Doppler effect does not usually affect the receiver. The stationary nature of VLC-based vehicular channel has been studied in [6] with better results than in DSCR. The probability of having a coherence time on the range of hundreds of milliseconds, for vehicular VLC links in urban scenarios is about $90 \%$ [7].

An interesting VLC solution for vehicular systems has been proposed in [8], using Optical Camera Communication (OCC) to bring $\mathrm{V} 2 \mathrm{I} / \mathrm{I} 2 \mathrm{~V}$ and suggesting the frame format to be used. Drawbacks come from the low data rate and the processing necessities for the system, as the region of interest of the cameras will be changing frame to frame due to vehicle movement.

In this paper, the architecture of a VLC system for vehicular communication in tunnels is proposed. In addition, its feasibility is evaluated by simulations. The paper is organized as follows. Section II describes the system environment and topological considerations previously taken into account before implementing the system. Section III details the evaluation parameters and the employed methodology, whilst section IV discusses the results. Finally in Section V some conclusions are extracted.

\section{SySTEM OVERVIEW}

\section{A. Scenario Description}

A prototypical scenario for the simulations, based on the European regulations of lighting inside tunnels [9] has been designed. Vehicular tunnels on double-way roads have a height of $4.3 \mathrm{~m}$ to $4.9 \mathrm{~m}$ a width of $13.2 \mathrm{~m}$ to $15 \mathrm{~m}$ width for the vehicular road and sidewalks. Another relevant fact to be considered is the luminous intensity, which depends on the distance to tunnel's gates so as to allow drivers to have a soft transition to the solar light and avoid glare-caused accidents. According to the adaptation time of human eyes and the distance travelled by the car (given by the recommended speed), the tunnel has been classified in different zones with a determined luminosity level. The most extended zone inside the tunnel is called the interior zone and has been selected for this work. The reason is because it is the part where the vehicle will spend more time. The average luminosity in that area is $4 \mathrm{~cd} / \mathrm{m}^{2}$, the maximum distance between LED lamps is 15 meters, and their height, 4 meters. Calculation of the average luminosity takes into account not only the LoS component, but the reflection on the asphalt area. The lamps considered for the simulations were commercial models. They are specially designed to provide an almost uniform light distribution with a built-in diffuser, reflectors and lens. Thus, their radiation pattern does not follow the typical Lambertian approach. The chosen commercial lamp to be simulated is the ARIANA from Saludes Lighting ${ }^{\circledR}$, with an electrical power of 150 watts, efficiency of $100 \mathrm{~lm} / \mathrm{W}$ and a color temperature of $4700 \mathrm{~K} \mathrm{[10]}$. The reflection values of different surfaces
TABLE I

REFLECTIVITIES OF THE TYPICAL MATERIALS USED IN TUNNELS [12] [13].

\begin{tabular}{ccc}
\hline Material & Reflection Pattern & Reflectivity \\
\hline Asphalt & Lambertian & 0.07 \\
Concrete & Lambertian & 0.4 \\
Road white paint & Phong & 0.5
\end{tabular}

on the tunnels can be consulted in [11]. For simplicity, as the reflection coefficient of clean asphalt is well below $10 \%$ in this model. The reflection values of the different tunnel materials considered for the simulated scenario can be observed in Table I.

\section{B. Architecture}

The use of VLC for communication inside tunnels has been previously proposed in the mining industry for location purposes [14] - [15] where the existence of volatile and flammable gases, and the bad propagation conditions makes unsuitable the use of RF communication technologies. The studies performed in [16] were focused on the path loss and time delay, but in this case a full duplex VLC system is needed for the V2I transmission.

The proposed system is formed by 3 types of modules. The first type is VLC Access Points (VLC-AP) which is part of the tunnel infrastructure and a group of this module will be distributed along the tunnel. The second is the Mobile Unit (MU) located on the vehicle. And finally, the Aggregation Agent (AA) which coordinates the VLC-APs.

VLC-AP and the MU are formed by 3 parts: a VLC transmitter, a VLC receiver and a Control Unit. Each VLCAP transmitter contains the circuity for the transmission and the power stage for the lamps of the tunnel. Unlike traditional transceivers that integrate transmitter and receiver within the same enclosure, the proposed architecture of VLC-AP separates them in order to take advantage of the car lighting. The receiver is located at a height of $1 \mathrm{~m}$, on a structure located on one side of the road. The main advantage of this design is the improvement of estimated signal reception for the uplink with respect to traditional VLC systems. Which they need additional technologies like Infrared (IR) vertical links for example. Moreover, significant changes on the vehicle front lamps would not be needed. The receiver uses a photodiode as a transducer, an Automatic Gain Control (AGC) circuit, and the circuitry for the signal demodulation. Both receiver and transmitter are connected through a fiber optic to the Control Unit. This unit is responsible to deal with the physical layer frame and will request retransmissions to the vehicle when the frame is corrupted depending on the application. The control unit of VLC-AP has communication with the AA. This unit evaluates VLC link parameters and the information from the vehicle to determinate when it is necessary to make a handover request to the $\mathrm{AA}$.

Mobile Unit is supposed to have a receiver located on the hood of the car, while headlights are used as transmitter, it 
has similar elements respect to the VLC-AP. The control unit of the MU can be integrated in the on-board computer, using the CAN BUS [17] integrated on the car for connecting the MU elements. With the on-board computer, the MU could obtain some vehicle's internal status and communicates to the computer crucial information from third parties. The uses of CAN BUS is in order to do not modify the ITS architecture, but it does not imply the VLC link transmit with this protocol.

The AA is the system element that enables the connection among the different VLC-APs on the tunnel and the core network. It manages the handover process of the vehicles when they are crossing the tunnel. Additionally it routes the packets inside the tunnel and decide which packets need to be sent thought the core network. The AA also connects with the higher communication network layers, as was proposed in [18]. Fig. 1 shows the topology for the different elements of the tunnel network.

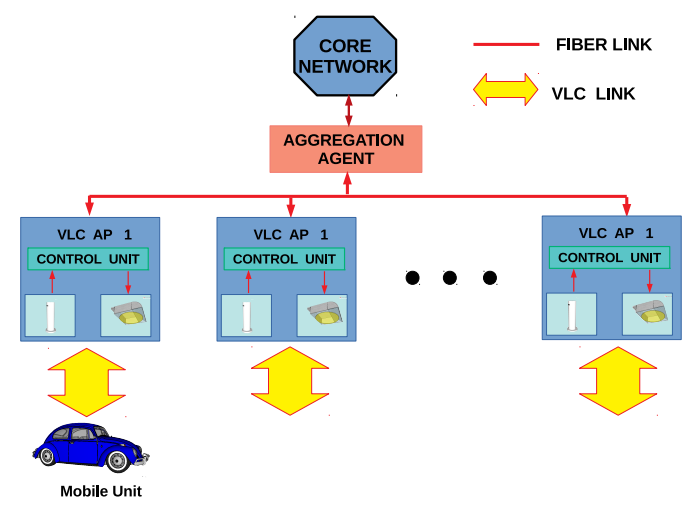

Fig. 1. Topology of the proposed system.

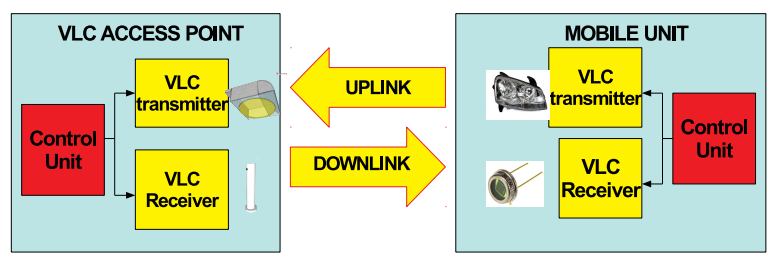

Fig. 2. VLC link between VLC AP and mobile unit.

Fig. 1 depicts the representation of the proposed system architecture while the block diagram of the VLC communication system is shown in Fig. 2.

The system design enables that the MU can provide information to the system in order to improve handover mechanisms. The mobile unit gets the vehicle's internal information via CAN BUS, and this information is given as feedback to get a better estimation when the handover will be required. Information like could be speed, the direction of the car or acceleration. Furthermore, as the tunnel is a confined scenario, it simplifies the localization problem. Other useful information can be obtained by the VLC-AP, as can be the Received
Signal Strength (RSS) from the vehicle. Fig. 3 shows the representation of the proposed system architecture.

This work is the initial stage of the research project. In this, the channel properties are studied assuming as Time-Invariant System. The studied optical communication system's goal is to satisfy at least the minimum requirement of ITS. The ITS applications based on DSCR present an average data rate of $6 \mathrm{Mbps}$ (maximum $12 \mathrm{Mbps}$ ), but the actual transfer speed highly depends on the scenario's conditions. On the other hand, The messages of ITS must have $100 \mathrm{~ms}$ of latency in the case of not vital messages, and their length is from 204 to 904 bits [19]. The platooning's data rate requirements are lower, only100 kbps are needed [20].

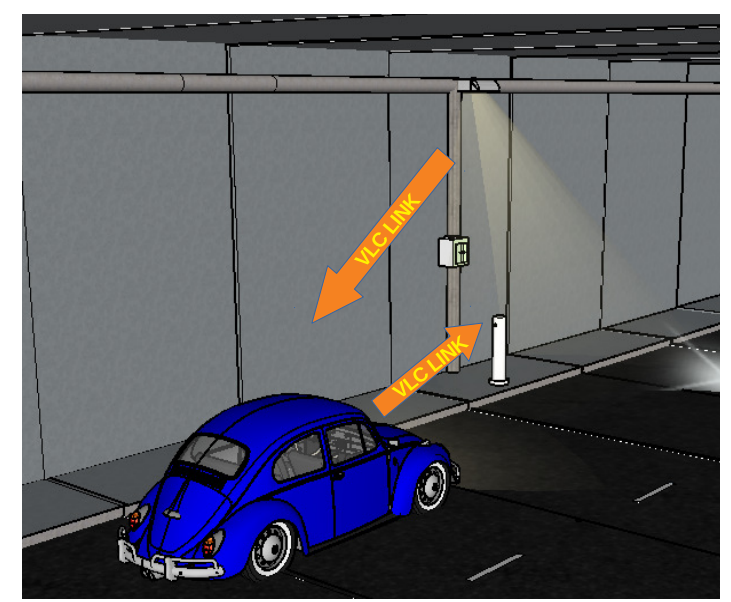

Fig. 3. Vehicular Full-Duplex VLC communications system for tunnels.

\section{Performance EVAluation}

In this paper only the downlink will be evaluated, because its analysis shows the feasibility of the system. The link behaviour depends on the illumination facilities characteristics and deployment, which are not allowed to be modified. The main aspects to evaluate are the links coverage areas in the worst cases and the maximum possible channel capacity. The maximum channel capacity results do not provide a realistic description of the system, since it only considers the effects of the propagation medium. There exist other aspects to be considered for realistic evaluation, such as the LEDs maximum commutation frequency, the driver circuitry frequency response, or the reception system in vehicles, etc. Additionally, some external agents degrade the channel for example, smog from vehicles, dust or even the vibration caused by the movement of great numbers of cars. These effects were not included in this channel evaluation, their impact on the channel will be studied in the future. Nevertheless, we only consider the channel impulse response (CIR) h(t), corresponding to the described scenario as a way to estimate the channel capacity. Furthermore, inside the CIR, the DC channel gain HO will serve as an indication of the maximum reachable Signal-toNoise Ratio (SNR). The main noise sources to be considered in the SNR estimation are: background noise produce for 
light sources external to the system, shot noise caused by the photodetector and the thermal noise caused by the electrical preamplifier. In this work, the contribution of background noise can be neglected because of the scenario configuration where the system is confined from the sun or other external lights sources. As the lights in the tunnel are part of the system, they will be better considered as interference sources instead of noise ones.

The Capacity of the channel, following the well-known Shannon approach, is related to the bandwidth and Noise Spectral Density N0, which depends on the selected receiver transducer. Unless in most cases it will be based on PIN photodide, in case of using an Avalanche Photodiode the calculation of N0 will be slightly different. Channel Impulse Response (CIR), will be obtained through a Modified Ray Tracing Monte Carlo (MMC) simulation has been employed [19]. This model will require a complete scenario description, as well as the emitter and the receiver characteristics as initial data for the calculations. Then, $\mathrm{Nr}$ rays are generated with random direction, and their trajectories and impact point will be calculated and a new ray with a random direction is generated. In all reflections a deterministic lighting component is obtained so as to shorten the simulation time. When rays finally reach the receiver, all the contribution are stored with an arrival time information. As it was previously stated, the main motivation of this paper is studying the coverage in terms of DC Channel Gain in different points of the scenario.

The simulation scenario is a tunnel with a dimension of 14.2 meters width, 4.9 meters high and 15 meters long. The tunnel model was built in SketchUp software, while the channel simulator was implemented in Python. In this scenario only one lamp will be simulated, it is located in the following point: 7.1 meters witdh, 7.5 meters stretch, and 4 meters high, taking the corner of the enter of the gate as reference point. The normal vector of the lamp is on the $\mathrm{z}$ axis, in direction of the floor. The area was selected to be the maximum area that the Access Point will provide service. The receivers will be a group of 625 . The receivers are uniformed distributed in a grid of $25 \times 25$ across the scenario.

Lamp ARIANA was chosen for the simulation. According to its technical datasheet has the radiation pattern, this pattern was recreated approximating the values. The recreation pattern can be seen in Fig. 4 . The receiver for the evaluation is a photodiode with a receiver area of $7.5 \mathrm{~mm}^{2}$ and Field of View of $68^{\circ}$.

The system configuration in this scenario causes that it has minimum Co-Channel Interference (CCI). Firstly the different VLC-AP downlinks in the interior zone of the tunnel, do not have any overlapping area. In case of other tunnel zones where exist overlapping, the implementation of a MAC protocol will reduce CCI. Furthermore, the headlight from the vehicle has descent radiation pattern [21], which means that MU receiver cannot direct contribution from other vehicles headlamps. In a similar situation, view of VLC-AP receivers in the tunnel side walls avoids the reception of signals from VLC-AP transmitter, since there is no LoS with the receiver. In the simulations, this contribution has values below $100 d B$. In the case of the multiple vehicle transmissions to the same VLC-AP, a MAC protocol needs to be designed and implemented to avoid interferences.

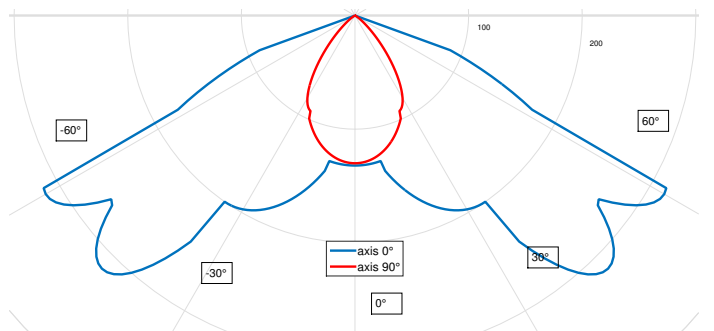

Fig. 4. Approximation of the radiation Pattern of ARIANA lamp.

\section{RESUlTS}

In this section the results will be discussed. Firstly, the DC Channel Gain will be analysed, focusing on its distribution in the simulated area. Results presented in Fig.5, correspond to the scenario where a VLC-AP has to cover maximum permissible area, the lamp in it is installed on the center of the tunnel. From this setup, results show that the total area is not totally covered for that lamp positioning. In the case of the lamps installed on the tunnel's walls, there will be 2 lamps on each tunnel segment, for this reason the lamp only has to span the half of simulated area.

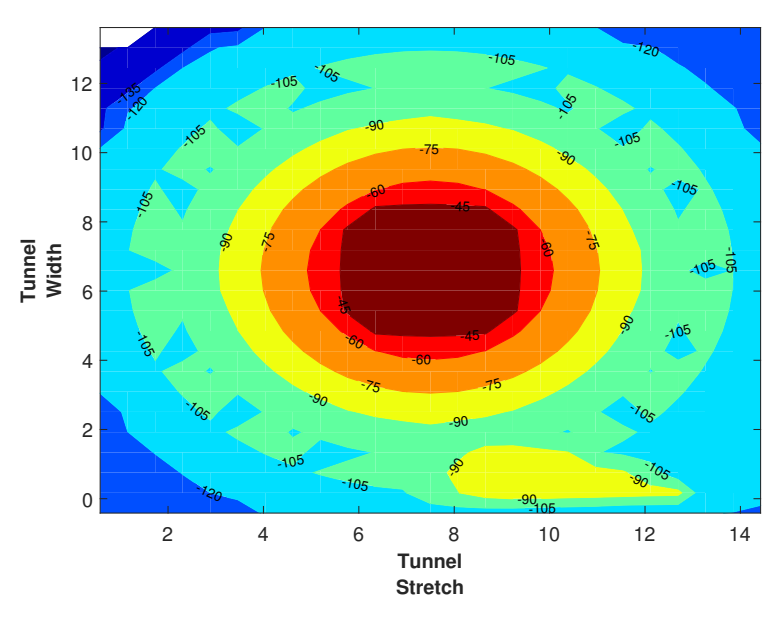

Fig. 5. DC channel Gain on tunnel area at 1 meter of height.

The cover area is better displayed in Fig 5, where it can be appreciated that the highest channel gain value is $-37.41 \mathrm{~dB}$. A more detailed presentation of the data is shown on Fig 6 .

Another evaluated parameter is the theoretical channel capacity, which is display on Fig.7. The figure displays high data rate values concentrated in a narrow area in the centre. In the evaluation of both parameters, the reflection from the vehicle surfaces, or other vehicles reflections has not been taken in consideration. In future works this assumption will be studied for more accurate CIR estimations. 


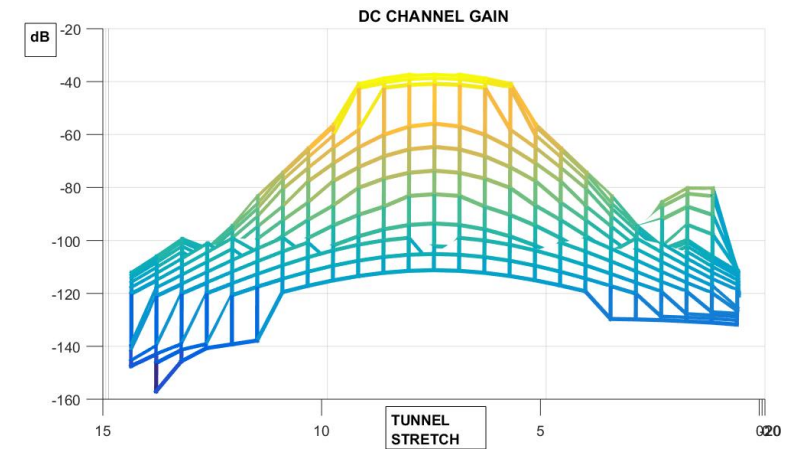

Fig. 6. DC channel Gain on tunnel area at 1 meter of height. On the top the $\mathrm{X}$ axis (stretch) view.

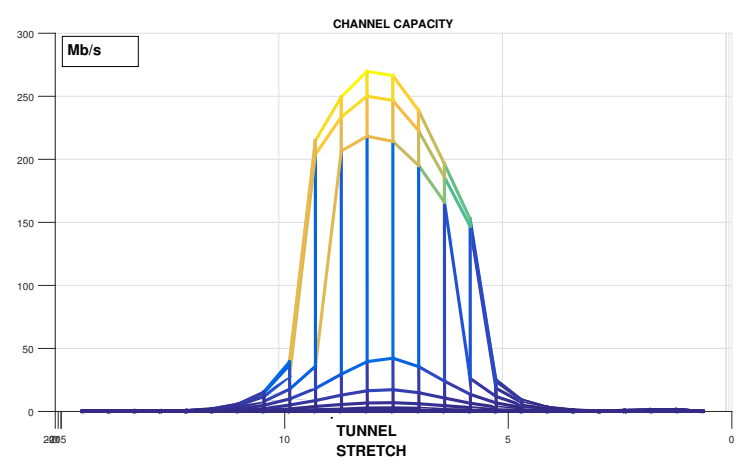

Fig. 7. Channel capacity in Mbps, on the axis X (stretch).

Relation between the receiver power and DC channel gain is given by the Eq. 1. As can be seen, it depends on the emitted optical power, but in the most of commercial lamps the technical information does not provide the optical power value. To obtain the lamp optical power is necessary to know among other things, the spectral power distribution. In [22] there is a database of spectral power distribution of different types of lamps. However, for the employed lamp does not exist that information, thus a temperature colour approximation was used. After the conversion, the lamp power is 41.78 watts.

$$
P_{r}=P_{t} * H(0)
$$

As it was commented before, the lamps are designed to be located on the walls of tunnel. Fig 9 shows the propagation taking in consideration the position of the lamp next to the tunnel wall, with a distance of $20 \mathrm{~cm}$. In the figure can be seen a greater area distribution in comparison with that corresponding to the lamp located at the centre of the tunnel, but the receiver power decreased until a maximum value of $-39.97 d B$.

\section{Conclusion}

In this paper, a communication system for vehicles in tunnels based on VLC was proposed. The system's uplink CIR

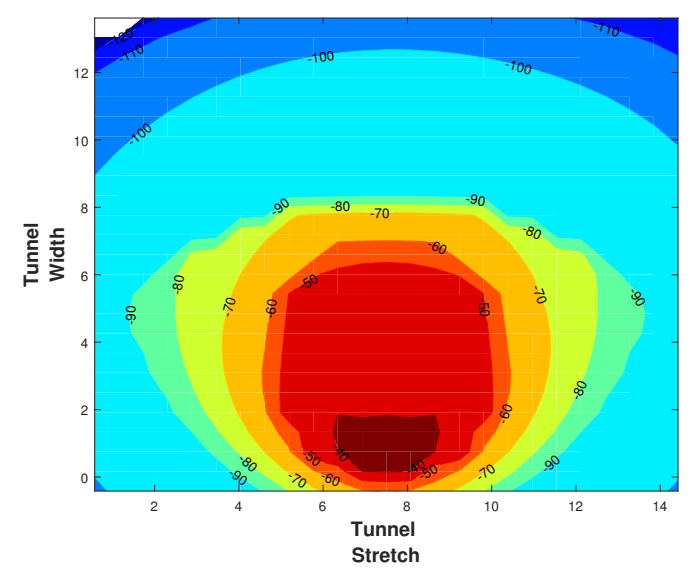

Fig. 8. DC channel Gain on tunnel area at 1 meter of hight. Lamp at 20 centimeters of the wall and $30^{\circ}$. On the top the $\mathrm{X}$ axis (stretch) view, on the bottom $\mathrm{Y}$ axis (width) view.

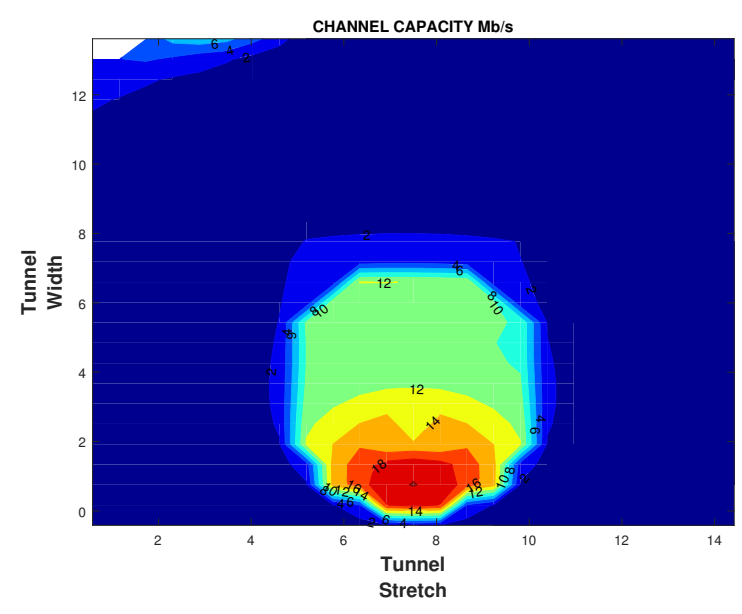

Fig. 9. Channel Capacity on tunnel area at 1 meter of hight. Lamp at 20 centimeters of the wall and $30^{\circ}$. On the top the $\mathrm{X}$ axis (stretch) view, on the bottom $\mathrm{Y}$ axis (width) view.

have been calculated in order to determine the feasibility of the system. On the results show first, the total area per AP is not totally covered. Secondly, high signal strength levels are concentrated in $8 \times 8$ meters area. On contrary for a lamp lateral position scenario, full coverage is got, but with lower optical power. Simulations results also show a channel capacity of over $100 \mathrm{Mbps}$, in great part area. And at some point really high values until close to $300 \mathrm{Mbps}$. This is a really high value, but it is a theoretical upper bound, as is explained below.

The present work is an initial stage evaluation of this kind of applications (VLC in tunnels). More complex simulations need to be done, in which the channel will be studied as Time Variant System to determinate the maximum possible frame size, the link duration and other information more. Besides the external agents presents inside a tunnel that affects the VLC channel need to be studied and included on the channel 
evaluations. External agents like can be the impact of the vibration caused by the vehicle movements, the reflection of other vehicles, pollution agents(smoke, dust,etc) and others.

Another future work is the uplink analysis. For this task is necessary to get enough information about the radiation pattern of the vehicle. With both links information the performance of the system can be predicted, and potential risks. Also, is necessary to evaluate the CCI caused by vehicles that are communicating with the same VLC-AP.

Finally, the simulations were done assuming a straight part of the tunnel, both links will be affected in a zone of the tunnel where a curve exists. With the gather information, more detailed system model will be designed. The main goals of the research line are studied and made a contribution in Link and Network Layer.

\section{ACKNOWLEDGMENT}

This work has been funded in part by the EU, program MSCA, ITN VISION 764461, and the Spanish Ministry of Research and innovation, Project OSCAR TEC2017-84065C3-1-R.

\section{REFERENCES}

[1] G. Karagiannis, O. Altintas, E. Ekici, G. Heijenk, B. Jarupan, K. Lin, and T. Weil. Vehicular networking: A survey and tutorial on requirements, architectures, challenges, standards and solutions. IEEE Communications Surveys Tutorials, 13(4):584-616, Fourth 2011.

[2] J. B. Kenney. Dedicated short-range communications (dsrc) standards in the united states. Proceedings of the IEEE, 99(7):1162-1182, July 2011.

[3] A. Hrovat, K. Guan, and T. Javornik. Traffic impact on radio wave propagation at millimeter-wave band in tunnels for $5 \mathrm{~g}$ communications. In 2017 11th European Conference on Antennas and Propagation (EUCAP), pages 2903-2906, March 2017.

[4] H. Viittala, S. Soderi, J. Saloranta, M. Hamalainen, and J. Iinatti. An experimental evaluation of wifi-based vehicle-to-vehicle (v2v) communication in a tunnel. In 2013 IEEE 77th Vehicular Technology Conference (VTC Spring), pages 1-5, June 2013.

[5] L. Bernado, A. Roma, A. Paier, T. Zemen, N. Czink, J. Karedal, A. Thiel, F. Tufvesson, A. F. Molisch, and C. F. Mecklenbrauker. In-tunnel vehicular radio channel characterization. In 2011 IEEE 73rd Vehicular Technology Conference (VTC Spring), pages 1-5, May 2011.

[6] Z. Cui, C. Wang, and H. Tsai. Characterizing channel fading in vehicular visible light communications with video data. In 2014 IEEE Vehicular Networking Conference (VNC), pages 226-229, Dec 2014.

[7] L. Wu and H. Tsai. Modeling vehicle-to-vehicle visible light communication link duration with empirical data. In 2013 IEEE Globecom Workshops (GC Wkshps), pages 1103-1109, Dec 2013.

[8] and. Handover in outdoor visible light communication system. In 2017 International Conference on Information Networking (ICOIN), pages 67-69, Jan 2017.

[9] Dirección General de Industria, Energía y Minas de Madrid. Guia de iluminación en tuneles e infraestructuras subterraneas. Standard, Consejería de Economía y Hacienda de Madrid, Madrid, Spain, 2015.

[10] Saludes Lighting. ARIANA Luminaria LED para tuneles, 2015. -.

[11] B. W. Kim and S. Jung. Vehicle positioning scheme using v2v and v2i visible light communications. In 2016 IEEE 83rd Vehicular Technology Conference (VTC Spring), pages 1-5, May 2016.

[12] Dirección General de Industria, Energía y Minas de Madrid. Guia técnica de aplicación de eficiencia en iluminación de alumbrado exterior-anexo ii. Standard, Consejería de Economía y Hacienda de Madrid, Madrid, Spain, 2013.

[13] Martin Herold. Understanding spectral characteristics of asphalt roads. Available at http://www.geogr.uni-jena.de (2019/04/3).

[14] D. Iturralde, F. Seguel, I. Soto, C. Azurdia, and S. Khan. A new vlc system for localization in underground mining tunnels. IEEE Latin America Transactions, 15(4):581-587, April 2017.
[15] N. Krommenacker, . C. Vsquez, M. D. Alfaro, and I. Soto. A selfadaptive cell-id positioning system based on visible light communications in underground mines. In 2016 IEEE International Conference on Automatica (ICA-ACCA), pages 1-7, Oct 2016.

[16] J. Wang, A. Al-Kinani, W. Zhang, C. Wang, and L. Zhou. A general channel model for visible light communications in underground mines. China Communications, 15(9):95-105, Sep. 2018.

[17] V. Matus, C. A. Azurdia-Meza, S. Cspedes, P. Ortega, S. MontejoSnchez, J. Rojas, and I. Soto. Implementation of a low-cost vehicular vlc system and can bus interface. In 2018 11th International Symposium on Communication Systems, Networks Digital Signal Processing (CSNDSP), pages 1-5, July 2018.

[18] C. Kim, S. Choi, and S. Koh. Idmp-vlc: Iot device management protocol in visible light communication networks. In 2017 19th International Conference on Advanced Communication Technology (ICACT), pages 578-583, Feb 2017.

[19] A. Cilean and M. Dimian. Current challenges for visible light communications usage in vehicle applications: A survey. IEEE Communications Surveys Tutorials, 19(4):2681-2703, Fourthquarter 2017.

[20] Max Schettler, Agon Memedi, and Falko Dressler. Deeply integrating visible light and radio communication for ultra-high reliable platooning. In WONS, 2019.

[21] Julio Chaves Pablo Benitez Juan C. Minano Oliver Dross, Aleksandra Cvetkovic. Led headlight architecture that creates a high quality beam pattern independent of led shortcomings, 2005.

[22] Lspdd: Lamp spectral power distribution database. http://galileo.graphycs.cegepsherbrooke.qc.ca/app/en/home. Accessed: 2019-04-06. 